Open Access

\title{
Dipeptide repeat protein inclusions are rare in the spinal cord and almost absent from motor neurons in C9ORF72 mutant amyotrophic lateral sclerosis and are unlikely to cause their degeneration
}

Jorge Gomez-Deza', Youn-bok Lee', Claire Troakes ${ }^{1,2}$, Matthew Nolan², Safa Al-Sarraj², Jean-Marc Gallo and Christopher E. Shaw ${ }^{1 *}$

\begin{abstract}
Introduction: Cytoplasmic TDP-43 inclusions are the pathological hallmark of amyotrophic lateral sclerosis (ALS) and tau-negative frontotemporal lobar dementia (FTLD). The $G_{4} C_{2}$ repeat mutation in C9ORF72 is the most common cause of ALS and FTLD in which, in addition to TDP-43 inclusions, five different di-peptide repeat (DPR) proteins have been identified. Di-peptide repeat proteins are translated in a non-canonical fashion from sense and antisense transcripts of the $\mathrm{G}_{4} \mathrm{C}_{2}$ repeat (GP, GA, GR, PA, PR). DPR inclusions are abundant in the cerebellum, as well as in the frontal and temporal lobes of ALS and FTLD patients and some are neurotoxic in a range of cellular and animal models, implying that DPR aggregation directly contributes to disease pathogenesis. Here we sought to quantify inclusions for each DPR and TDP-43 in ALS cases with and without the C9ORF72 mutation. We characterised the abundance of DPRs and their cellular location and compared this to cytoplasmic TDP-43 inclusions in order to explore the role of each inclusion in lower motor neuron degeneration.

Results: Spinal cord sections from ten cases positive for the C9ORF72 repeat expansion (ALS-C9+ve) and five cases that were not were probed by double immunofluorescence staining for individual DPRs and TDP-43. Inclusions immunoreactive for each of the DPRs were present in the spinal cord but they were rare or very rare in abundance (in descending order of frequency: GA, GP, GR, PA and PR). TDP-43 cytoplasmic inclusions were 45- to 750-fold more frequent than any DPR, and fewer than $4 \%$ of DPR inclusions colocalized with TDP-43 inclusions. In motor neurons, a single cytoplasmic DPR inclusion was detected (0.1 \%) in contrast to the $34 \%$ of motor neurons that contained cytoplasmic TDP-43 inclusions. Furthermore, the number of TDP-43 inclusions in ALS cases with and without the C9ORF72 mutation was nearly identical.

Conclusions: For all other neurodegenerative diseases, the neurotoxic protein aggregates are detected in the affected population of neurons. TDP-43 cytoplasmic aggregation is the dominant feature of ALS spinal cord pathology irrespective of C9ORF72 mutation status. The near absence of DPR inclusions in spinal cord motor neurons challenges their contribution to lower motor neuron degeneration in ALS-C9+ve cases.
\end{abstract}

Keywords: C9ORF72, Dipeptide repeat, TDP-43, Motor neuron, Amyotrophic lateral sclerosis

\footnotetext{
* Correspondence: Christopher.shaw@kcl.ac.uk

'Department of Basic and Clinical Neurosciences, Maurice Wohl Clinical

Neurosciences Institute, Institute of Psychiatry, Psychology and Neuroscience,

Kings College London, London SE5 8AF, UK

Full list of author information is available at the end of the article
} 


\section{Introduction}

Amyotrophic lateral sclerosis (ALS) is a progressive neurodegenerative disease characterised by the loss of motor neurons in the brain and spinal cord causing progressive paralysis of limb and bulbar function and death due to respiratory failure within an average of 3 years from symptom onset. Cytoplasmic inclusions containing the transactive response DNA-binding protein (TDP-43) are the neuropathological hallmark of $\sim 95 \%$ of ALS cases and $\sim 60 \%$ of frontotemporal dementia (FTD) cases [1]. Expansion of an intronic hexanucleotide repeat $\left(\mathrm{G}_{4} \mathrm{C}_{2}\right)$ in chromosome 9 open reading frame 72 (C9ORF72) is the most common genetic cause of ALS in people of European ancestry [2-4]. The number of pathological repeats varies from $300-3000$ in ALS cases and contrasts with up to 30 repeats found in healthy controls.

The neurotoxic mechanisms of the C9ORF72 expansion are unknown but three mechanisms have been proposed: loss of C9ORF72 protein function; toxicity due to RNA aggregation and toxicity due to repeat associated non-ATG (RAN) translation of dipeptide repeat (DPR) proteins. Although the $\mathrm{G}_{4} \mathrm{C}_{2}$ expansion does cause a decrease in C9ORF72 mRNA, it is not clear that this leads to loss of the C9ORF72 protein and no null alleles or missense mutations in C9ORF72 have been identified in ALS or FTD cases to support the loss of C9ORF72 protein function hypothesis. We, and others have shown that $\mathrm{G}_{4} \mathrm{C}_{2}$ repeats generate length-dependant RNase-resistant intranuclear RNA foci, which can sequester RNA binding proteins potentially leading to defective RNA processing [5]. Finally, the $\mathrm{G}_{4} \mathrm{C}_{2}$ repeat RNA form stable G-quadruplex secondary structures that can recruit the translation machinery [6] and generate five different DPRs; poly-GP, poly-GA and poly-GR from the sense $\mathrm{G}_{4} \mathrm{C}_{2}$ repeat strand and poly-PR, poly-PA and poly-GP from the antisense $\mathrm{G}_{2} \mathrm{C}_{4}$ repeat strand $[7,8]$. This last hypothesis has recently garnered much attention and is the one we wish to test in the context of human ALS tissues.

Antibodies to specific DPR proteins detect cytoplasmic aggregates of poly-GP, poly-GA and poly-PA and intranuclear aggregates of poly-PR and poly-GR in the cerebellum and frontal cortex of ALS and FTD patients $[9,10]$. DPR protein aggregates colocalize with p62 but not TDP-43 $[9,10]$ and the connection between the C9ORF72 mutation, DPR protein deposition and TDP-43 mislocalization is unknown. In the present study, we conducted a detailed comparison of the abundance and cellular location of the five DPR proteins and TDP-43 in the spinal cord of ten ALS-C9+ve cases. Using double immunofluorescence staining, we show that DPR protein aggregates are uncommon in the spinal cord of ALS-C9+ve cases, rarely co-localise with TDP-43, and are almost absent from motor neurons. The abundance of TDP-43 inclusions in ALS cases with or without a C9ORF72 repeat expansion was broadly similar. Given that TDP-43 mislocalization is a recognised cause of lower motor neuron neurodegeneration, and given the lack of DPRs in motor neurons, it is difficult to implicate DPRs in this process.

\section{Materials and methods \\ Cases}

A total of 30 spinal cord sections were analysed for the presence of TDP-43 and all DPR inclusions by double immunofluorescence from ten ALS cases positive for C9ORF72 intronic expansion (ALS-C9+ve) and 13 sections from five ALS cases negative for C9ORF72, FUS and SOD1 mutations (365 sections in total). Sections of frontal lobe and cerebellar cortex from a case of C9FTD with abundant p62 pathology were chosen as a positive control for DPR staining. All cases were provided by the Medical Research Council London Neurodegenerative Diseases Brain Bank (Institute of Psychiatry, Psychology and Neuroscience, King's College London). Samples were collected and distributed in accordance with local and national research ethics committee approvals. Expansion carriers were identified using repeat primed PCR [4] and all of the cases had previously been reported to show characteristic cerebellar p62 and TDP-43 pathology [11,12]. Details of the age, sex and post-mortem delay are recorded in Table 1 and show little difference between cases with and without C9ORF72 mutations.

Table 1 Clinical Data of cases studied

\begin{tabular}{|c|c|c|c|c|}
\hline Case & Age & Sex & PMD & Diagnosis \\
\hline 1 & 73 & $M$ & 42 & ALS-TDP \\
\hline 2 & 70 & $\mathrm{~F}$ & 27 & ALS-TDP \\
\hline 3 & 60 & M & 70 & ALS-TDP \\
\hline 4 & 44 & $\mathrm{~F}$ & 24 & ALS-TDP \\
\hline 5 & 68 & M & 5 & ALS-TDP \\
\hline mean \pm SEM & $63 \pm 5$ & & $33 \pm 11$ & ALS-TDP \\
\hline 6 & 59 & $\mathrm{~F}$ & 35 & ALS-C9 + ve \\
\hline 7 & 70 & M & 38 & ALS-C9 + ve \\
\hline 8 & 59 & M & 46 & ALS-C9 + ve \\
\hline 9 & 43 & $F$ & 69 & ALS-C9 + ve \\
\hline 10 & 53 & M & 82 & ALS-C9+ve \\
\hline 11 & 70 & M & 60 & ALS-C9 + ve \\
\hline 12 & 55 & M & 76 & ALS-C9 + ve \\
\hline 13 & 58 & M & 11 & ALS-C9 + ve \\
\hline 14 & 64 & M & 68 & ALS-C9 + ve \\
\hline 15 & 51 & M & 64 & ALS-C9 + ve \\
\hline mean \pm SEM & $58 \pm 2$ & & $55 \pm 5$ & \\
\hline
\end{tabular}

$P M D$ post mortem delay (hours) 


\section{Motor neuron counting and double label immunofluorescence}

Histological examination was performed on $7 \mu \mathrm{m}$ sections prepared from formalin-fixed, paraffin -embedded tissue from spinal cords of all ALS cases. Sections were stained with hematoxylin and eosin to perform motor neuron counts, and sequential sections were processed for double label immunofluorescence for the DPRs and TDP-43. Prior to double immunofluorescence staining, paraffin was removed with xylene and all sections were rehydrated in an ethanol series (100, 95, and $70 \%$ ) for 3 min per step. Slides were incubated in $0.3 \%$ Sudan black for 5 min to quench autofluorescence and washed with water. Antigen retrieval was carried out by microwaving for $6 \mathrm{~min}$ at maximum power and $12 \mathrm{~min}$ at medium power in $100 \mathrm{mM}$ sodium citrate buffer ( $\mathrm{pH}$ 6.0). Non-specific binding sites were then blocked for 20 min using $5 \%$ normal donkey serum in PBS.

For double immunofluorescence staining, spinal cord sections were incubated with primary rabbit antibodies against the five different DPRs (poly-GA, GP, GR, PR and PA) at a dilution of 1:100 together with TDP-43 (rat monoclonal TDP-43 Sigma Scientific (SIG-39850)) at a dilution of $1: 100$ overnight in a humid chamber at $4{ }^{\circ} \mathrm{C}$. The DPR antibodies were obtained from Dr. Leonard Petrucelli and colleagues and were previously characterised [13]. After 3 washes with PBS, sections were incubated with anti-rabbit (Alexa Fluor 594) and anti-rat (Alexa Fluor 488) secondary antibodies for $1 \mathrm{~h}$ at room temperature. DAPI (Sigma) was used to counterstain nuclei. Sections were mounted in Fluorsave. Semiquantitative and quantitative evaluation of DPR and TDP-43 pathology was performed using Zeiss Axiovert S100 microscope. Aggregates were also imaged using a Leica Confocal SP microscope.

\section{Semi quantitative and quantitative evaluation of pathology}

The presence of DPR and TDP-43 inclusions were scored in the anterior horn of the spinal cord using a previously published semi-quantitative grading scale [14], in which the total number of immunoreactive inclusions, as well as the cytoplasmic inclusions (CI) and the intranuclear inclusions $(\mathrm{NI})$, were rated as follows: 0 - Absent, 0.5 - one or two inclusions in the whole section, 1- very few, 2- occasionaleasy to find and a few cells are affected, 3- moderate- many of the cells are affected, inclusions are easy to find, 4numerous- nearly all of the cells are affected. Additionally, each section was re-analysed and the number of DPR and TDP-43 inclusions was counted manually in a systematic, blinded manner. The number of DPR aggregates was counted per section and averaged per case. Statistical analysis was performed with GraphPad Prism software (version 5.0).

\section{Results}

Appearance of DPR proteins in ALS spinal cords

The appearance and subcellular location of each type of DPR inclusion within the spinal cord was recorded (Fig. 1a-h). Poly-GA aggregates were the most abundant DPR detected with cytoplasmic immunoreactive inclusions more common than intranuclear inclusions. This is consistent with previous studies in FTD and ALS [9]. Cytoplasmic inclusions were characteristically large, irregular and perinuclear whilst nuclear inclusions were much smaller and round. Poly-GP inclusions, the second most common DPR inclusion, were all cytoplasmic and in a perinuclear position (Fig. 1b). We observed very few cytoplasmic poly-GR inclusions and only one small intranuclear aggregate. Poly-PR cytoplasmic aggregates were also both nuclear and cytoplasmic (Fig. 1e, g). Similarly, we were able to identify a total of only seven poly-PA inclusions (six cytoplasmic and one nuclear; Fig. 1d, h) and four poly-PR inclusions (two cytoplasmic and two nuclear; Fig. 1e, g) inclusions in all the analyzed cases (Fig. 1d, h). No immunoreactive DPR aggregates were identified in any C9 negative ALS TDP-43 cases confirming the mutation-specificity of these inclusions and validating the antibodies. In a small number of cells that contained DPR aggregates, granular TDP-43 staining was seen in the cytoplasm or absent in the nucleus (Fig. 1) consistent with previous studies [9]. This pattern of TDP-43 staining was however much more common in neurons without DPR aggregates.

\section{All DPRs are rare and some very rare, but TDP-43 pathology was abundant and consistent}

The frequency of each DPR inclusion was initially scored using a semi-quantitative system for each case (Table 2). DPRs translated from the sense strand (poly-GP, poly-GA and poly-GR) were more abundant than those translated solely from the antisense strand (poly-PR, poly-PA). Although poly-GA and poly-GP were the most abundant immunoreactive inclusions (scoring of 0.9 and 0.7 , respectively) all DPRs were scored as either rare or very rare and there was not a single case that scored above 1.0 (rare) and no more than twelve inclusions of any DPR was observed in any of the cases analysed. No case scored above 0.5 (vary rare) for poly-GR, poly-PA and poly-PR inclusions and poly-GR and poly-PR inclusions were absent from several cases. The scoring of DPR aggregates within cases was highly consistent (poly-GA = $0.9 \pm 0.1$, poly-GP $=0.7 \pm 0.1$, poly-GR $=0.8 \pm 0.21$, poly$\mathrm{PA}=0.4 \pm 0.20$ and poly-PR $=0.3 \pm 0.1)$. Of note, there was no significant difference in the scoring of TDP-43 inclusions for ALS cases lacking the C9ORF72 mutation $(2.8 \pm 0.2)$ when compared to those carrying the C9ORF72 mutation $(2.9 \pm 0.1)$. Thus, the presence of DPR inclusions 


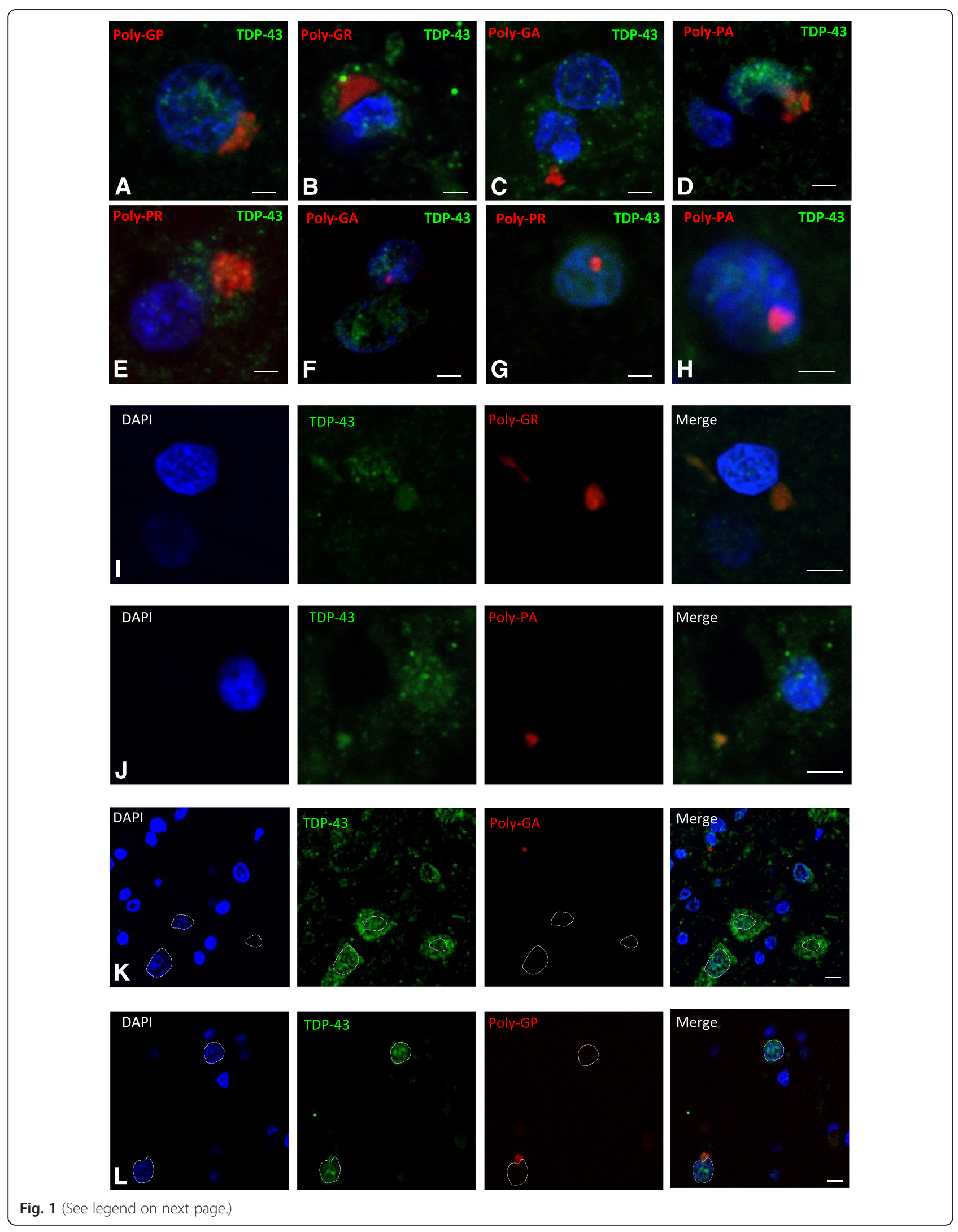


(See figure on previous page.)

Fig. 1 Representative images of DPR inclusions. $\mathbf{a}$-j are representative immunofluorescence confocal images of DPR (red) and TDP-43 (green) (scale bar $=20 \mu \mathrm{m}$ ). a-e show cytoplasmic DPR inclusions, which are often perinuclear. $\mathbf{f}-\mathbf{h}$ show nuclear DPR inclusions. $\mathbf{i}$ and $\mathbf{j}$ show evidence of DPR inclusions colocalizing with TDP-43. $\mathbf{k}$ and $\mathbf{I}$ are lower power images of the anterior horn showing the relative abundance of DPR and TDP-43 inclusions (scale bar $=50 \mu \mathrm{m}$ ). I shows a poly-GA inclusion, which is not in the motor neurons (dotted nucleus) that show extensive cytoplasmic TDP deposition. $\mathbf{k}$ shows a poly-GP inclusion within a motor neuron (identified by its large pale nucleus), which does not show cytoplasmic TDP-43 inclusions. Motor neurons were identified by their characteristic large pale nucleus (dotted line)

in the spinal cord does not appear to be correlated with the abundance of TDP-43 inclusions.

In order to provide greater detail, the number of TDP-43 aggregates in the anterior horn of the spinal cord in each case was quantified in representative sections to explore any correlation between the abundance of DPR and TDP-43 inclusions. An average of $122 \pm 8$ TDP-43 aggregates was identified per section per case, which is between 45 - and 750- fold greater than the number of any DPRs inclusions (Additional file 1: Table S1). These results, graphically depicted in Fig. 2, demonstrate that the abundance of TDP-43 cytoplasmic inclusions vastly outnumbers the number of DPR inclusions, singly or collectively (note that the $\mathrm{Y}$ axis scale is a logarithmic scale).

\section{DPR and TDP-43 inclusions occasionally colocalize}

Although all DPRs were rare or very rare, we did see TDP-43 colocalize with 4 of the 12 poly-GR inclusions and 2 of the 7 poly-PA inclusions (Fig. 1i, j). Interestingly, the poly-GR immunoreactive aggregates that did not colocalize were often surrounded by TDP-43 (Fig. 1b). None of the nuclear poly-GR and poly-PA intranuclear inclusions colocalized with TDP-43. We did not observe any poly-GP, poly-GA or poly-PR immunoreactive inclusions that colocalized with TDP-43 inclusions.

\section{DPRs are almost absent from motor neurons}

ALS is characterised by the loss of lower motor neurons in the anterior horn of the spinal cord. Motor neurons are readily identified as they have large cell bodies and

Table 2 TDP-43 and DPR scoring

\begin{tabular}{|c|c|c|c|c|c|c|c|c|c|c|c|c|c|c|c|c|c|}
\hline \multirow[b]{2}{*}{ case } & \multirow{2}{*}{$\begin{array}{l}\text { TDP-43 } \\
\text { agg. }\end{array}$} & \multicolumn{3}{|c|}{ Poly-GP } & \multicolumn{3}{|c|}{ Poly-GA } & \multicolumn{3}{|c|}{ Poly-GR } & \multicolumn{3}{|c|}{ Poly-PR } & \multicolumn{3}{|c|}{ Poly-PA } & \multirow[t]{2}{*}{ Diagnosis } \\
\hline & & Cyt. & Nuc. & Tot. & Cyt. & Nuc. & Tot. & Cyt. & Nuc. & Tot. & Cyt. & Nuc. & Tot. & Cyt. & Nuc. & Tot. & \\
\hline 1 & 3 & 0 & 0 & 0 & 0 & 0 & 0 & 0 & 0 & 0 & 0 & 0 & 0 & 0 & 0 & 0 & ALS-TDP \\
\hline 2 & 3 & 0 & 0 & 0 & 0 & 0 & 0 & 0 & 0 & 0 & 0 & 0 & 0 & 0 & 0 & 0 & ALS-TDP \\
\hline 3 & 2 & 0 & 0 & 0 & 0 & 0 & 0 & 0 & 0 & 0 & 0 & 0 & 0 & 0 & 0 & 0 & ALS-TDP \\
\hline 4 & 3 & 0 & 0 & 0 & 0 & 0 & 0 & 0 & 0 & 0 & 0 & 0 & 0 & 0 & 0 & 0 & ALS-TDP \\
\hline 5 & 3 & 0 & 0 & 0 & 0 & 0 & 0 & 0 & 0 & 0 & 0 & 0 & 0 & 0 & 0 & 0 & ALS-TDP \\
\hline $\begin{array}{l}\text { MEAN } \\
\pm \\
\text { SEM }\end{array}$ & $2.8 \pm 0.2$ & & & & & & & & & & & & & & & & ALS-TDP \\
\hline 6 & 3 & 0.5 & 0 & 0.5 & 0 & 0 & 0 & 0.5 & 0 & 0.5 & 0 & 0 & 0 & 0.5 & 0 & 0.5 & ALS-C9 + ve \\
\hline 7 & 3 & 0.5 & 0 & 0.5 & 1 & 0.5 & 1.5 & 0 & 0 & 0 & 0 & 0 & 0 & 0 & 0 & 0 & ALS-C9 + ve \\
\hline 8 & 2 & 1 & 0 & 1 & 0.5 & 0 & 0.5 & 0.5 & 0 & 0.5 & 0 & 0 & 0 & 0.5 & 0 & 0.5 & ALS-C9 + ve \\
\hline 9 & 3 & 1 & 0 & 1 & 1 & 0.5 & 1.5 & 0 & 0 & 0 & 0 & 0 & 0 & 0.5 & 0 & 0.5 & ALS-C9 + ve \\
\hline 10 & 3 & 0.5 & 0 & 0.5 & 0.5 & 0.5 & 1 & 0.5 & 0 & 0.5 & 0 & 0 & 0 & 0 & 0 & 0 & ALS-C9 + ve \\
\hline 11 & 3 & 1 & 0 & 1 & 1 & 0.5 & 1.5 & 0 & 0.5 & 0.5 & 0.5 & 0 & 0.5 & 0.5 & 0 & 0.5 & ALS-C9 + ve \\
\hline 12 & 3 & 0.5 & 0 & 0.5 & 0.5 & 0 & 0.5 & 0.5 & 0 & 0.5 & 0.5 & 0.5 & 1 & 0 & 0 & 0 & ALS-C9 + ve \\
\hline 13 & 3 & 1 & 0 & 1 & 1 & 0.5 & 1.5 & 0.5 & 0 & 0.5 & 0.5 & 0 & 0.5 & 0.5 & 0 & 0 & ALS-C9 + ve \\
\hline 14 & 3 & 1 & 0 & 1 & 0.5 & 0 & 0.5 & 0 & 0 & 0 & 0 & 0 & 0 & 0 & 0 & 0 & ALS-C9 + ve \\
\hline 15 & 3 & 0.5 & 0 & 0.5 & 1 & 0 & 1 & 0 & 0 & 0 & 0 & 0 & 0 & 0 & 0 & 0 & ALS-C9 + ve \\
\hline $\begin{array}{l}\text { MEAN } \\
\pm \\
\text { SEM }\end{array}$ & $\begin{array}{l}2.9 \pm \\
1\end{array}$ & $\begin{array}{l}0.7 \pm \\
0.1\end{array}$ & 0.0 & $\begin{array}{l}0.7 \pm \\
0.1\end{array}$ & $\begin{array}{l}0.7 \pm \\
0.1\end{array}$ & $\begin{array}{l}0.2 \\
\pm 0\end{array}$ & $\begin{array}{l}0.9 \pm \\
0.1\end{array}$ & $\begin{array}{l}0.3 \pm \\
0.1\end{array}$ & $\begin{array}{l}0.0 \pm \\
0.1\end{array}$ & $\begin{array}{l}0.3 \pm \\
0.1\end{array}$ & $\begin{array}{l}0.1 \pm \\
0.1\end{array}$ & $\begin{array}{l}0.0 \pm \\
0.1\end{array}$ & $\begin{array}{l}0.2 \pm \\
0.1\end{array}$ & $\begin{array}{l}0.2 \pm \\
0.1\end{array}$ & $\begin{array}{l}0.0 \pm \\
0.1\end{array}$ & $\begin{array}{l}0.3 \pm \\
0.1\end{array}$ & \\
\hline
\end{tabular}




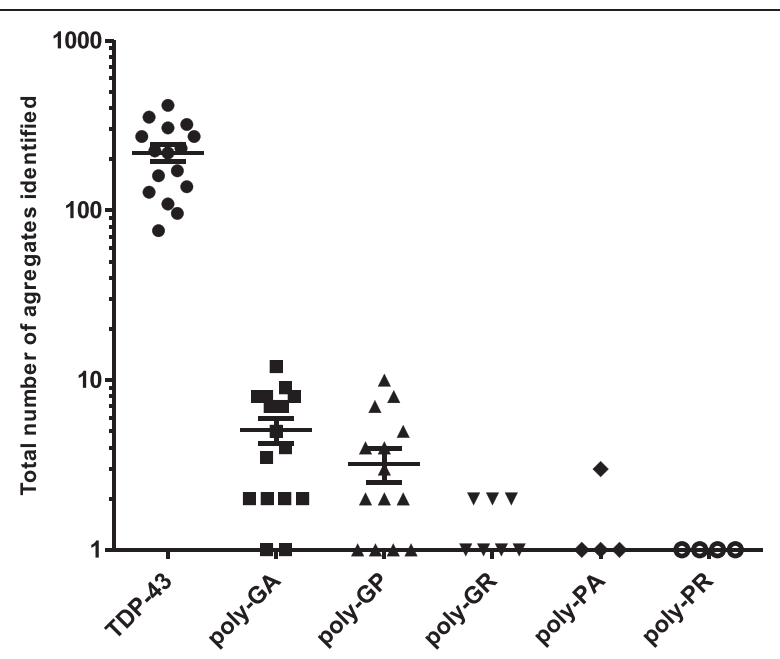

Fig. 2 Frequency of TDP-43 and DPR inclusion per case, per section. The number of TDP-43 and DPR immunoreactive inclusions was counted for each case and normalised per spinal cord section. The graph shows the number of TDP-43 and the five different aggregates in order of abundance. There are many more TDP-43 aggregates than any DPR. Note: The $Y$ axis scale is logarithmic scale. Horizontal bars show mean \pm SEM

large pale nuclei by H\&E staining and by DAPI for immunofluorescence. The total number of motor neurons was counted in order to assess the relative preservation of motor neurons between cases and identify any that contained TDP-43 and DPR immunoreactive inclusions. In total, of the 786 motor neurons analysed 268 (34\%) contained cytoplasmic TDP-43 inclusions (Fig. 1k), which is consistent with classical ALS pathology. In contrast, only one motor neuron contained a DPR inclusion, specifically a cytoplasmic poly-GP aggregate (0.1 \%) (Fig. 1l). Occasionally we observed DPR and TDP-43 aggregates in the same cell but never in motor neurons.

\section{Discussion}

The $\mathrm{G}_{4} \mathrm{C}_{2}$ repeat mutation in the C9ORF72 gene is the most common cause of ALS and FTLD known to date. A leading mechanistic hypothesis is that neurotoxicity is due to the expression and aggregation of DPRs, particularly poly-GR and poly-PR that form intranuclear inclusions [15-17]. In this study, we sought to characterise the abundance, subcellular and cellular localization of immunoreactive inclusions for each DPR and TDP-43 in the anterior horn of the spinal cord in C9ORF72 mutant positive and negative ALS cases.

Inclusions for all five DPRs were detected in the spinal cord of ALS-C9+ve cases but they were rare or very rare and it was uncommon to see DPR and TDP-43 inclusions in the same cell. Poly-GA inclusions, present in the cytoplasm and occasionally the nucleus, were the most common. Poly-GP inclusions were purely cytoplasmic, which is consistent with published reports [7$10,14,18,19]$. Of the five DPRs, only poly-GR and polyPA physically colocalized with TDP-43 inclusions, but this was a very rare event. The total number of TDP-43 inclusions in the spinal cord was between 45- and 750fold higher than the number of DPR inclusions. We detected only one motor neuron that contained a poly-GP inclusion, whereas on average $34 \%$ of motor neurons had cytoplasmic TDP-43 inclusions. Our results are consistent with those published using single staining techniques but are the first to use double labelling with TDP-43 and all five DPRs in the spinal cord of ALS cases [9]. We acknowledge that detection of DPRs by immunofluorescence imaging may be less sensitive than horse radish peroxidase (HRP) but these were abundant in the frontal cortex of our C9ORF72 mutation positive cases (Additional file 2: Figure S1).

Several groups have studied DPR protein toxicity in various cellular models but these have generated conflicting results [15-17,20,21]. Two recent reports describe the greatest toxicity from poly-GR and poly-PR expression in cells $[15,16]$ and the drosophila eye [22]. However, these models involve the overexpression of DPRs and no comparison was made with the burden of DPR deposition in human tissues. A recent study, in which $66 G_{4} C_{2}$ repeats were expressed in the central nervous system of mice, has shown that nuclear RNA foci, DPR inclusions and TDP-43 pathology were present in the cortex and hippocampus of mice, suggesting that the repeat itself, possibly through foci formation, RAN translation or another mechanisms entirely, caused aberrant TDP-43 deposition [23]. We have demonstrated that DPR inclusions are very rare in ALS-C9 +ve spinal cords, are infrequently associated with TDP43 inclusions and are almost absent from motor neurons. On this basis we find no pathological evidence that DPR aggregation contributes to lower motor neuron degeneration in ALS-C9+ve cases.

\section{Conclusions}

While we cannot entirely exclude the possibility that DPR aggregation and toxicity occurs at the submicroscopic level, through toxic DPR oligomers, or that all motor neurons containing DPR inclusions were lost, this explanation seems unlikely as neurotoxic protein aggregates are detected in the affected neurons for all other neurodegenerative diseases. As the number of TDP-43 inclusions in ALS cases, with and without the C9ORF72 mutation are nearly identical we can find no evidence that dipeptide repeat proteins are playing a pathogenic role in ALS-C9+ve cases. 


\section{Additional files}

Additional file 1: Table S1. Table showing the total numbers of TDP-43 and DPR aggregates per spinal cord section analysed.

Additional file 2: Figure S1. Representative images of DPR inclusions in the frontal cortex of C9ORF72 cases. Representative images of DPR aggregates in the frontal cortex of C9ORF72 mutation positive cases used as a positive control. Dotted area indicates the zoomed in area. Arrows indicate the presence of DPR aggregates. (Scale bar $=50 \mu \mathrm{m}$ ).

\section{Competing interests}

The authors declare that they have no competing interests.

\section{Authors' contributions}

JG-D and CES were responsible for the experimental design and paper writing. JG-D carried out the double immune fluorescence, quantification of aggregates and quantification of motor neurons. YL optimised the technique used in this study. CT, MN and SAS cut and prepared the spinal cord tissue used. J-MG and CES supervised the study and contributed to the data interpretation. All authors read and approved the final manuscript.

\section{Acknowledgements}

This research was principally supported by the King's Bioscience Institute and the Guy's and St Thomas' Charity Prize PhD Programme in Biomedical and Translational Science to JGD and a Strategic Grant Award from the Medical Research Council and the Wellcome Trust (grant reference 089701) to CES, with additional support from The Motor Neuron Disease Association, Heaton Ellis Trust, Psychiatry Research Trust and the American Amyotrophic Lateral Sclerosis Association. The institute of Psychiatry Psychology and Neuroscience Brain Bank receives funding from the Medical Research Council.

\section{Author details}

${ }^{1}$ Department of Basic and Clinical Neurosciences, Maurice Wohl Clinical Neurosciences Institute, Institute of Psychiatry, Psychology and Neuroscience, Kings College London, London SE5 8AF, UK. ${ }^{2}$ London Neurodegenerative Disease Brain Bank, Institute of Psychiatry, Psychology and Neuroscience, King's College London, London SE5 8AF, UK.

Received: 3 June 2015 Accepted: 12 June 2015

Published online: 25 June 2015

\section{References}

1. Neumann M et al (2006) Ubiquitinated TDP-43 in frontotemporal lobar degeneration and amyotrophic lateral sclerosis. Science 314(5796):130-3

2. Renton AE et al (2011) A hexanucleotide repeat expansion in C9ORF72 is the cause of chromosome 9p21-linked ALS-FTD. Neuron 72(2):257-68

3. DeJesus-Hernandez M et al (2011) Expanded GGGGCC hexanucleotide repeat in noncoding region of C9ORF72 causes chromosome 9p-linked FTD and ALS. Neuron 72(2):245-56

4. Smith BN et al (2013) The C9ORF72 expansion mutation is a common cause of ALS+/-FTD in Europe and has a single founder. Eur J Hum Genet 21(1):102-8

5. Lee YB et al (2013) Hexanucleotide repeats in ALS/FTD form lengthdependent RNA foci, sequester RNA binding proteins, and are neurotoxic. Cell Rep 5(5):1178-86

6. Zu T et al (2011) Non-ATG-initiated translation directed by microsatellite expansions. Proc Natl Acad Sci U S A 108(1):260-5

7. Mori Ket al (2013) The C9orf72 GGGGCC repeat is translated into aggregating dipeptide-repeat proteins in FTLD/ALS. Science 339(6125):1335-8

8. Ash PE et al (2013) Unconventional translation of C90RF72 GGGGCC expansion generates insoluble polypeptides specific to c9FTD/ALS. Neuron 77(4):639-46

9. Mackenzie IR et al (2013) Dipeptide repeat protein pathology in C9ORF72 mutation cases: clinico-pathological correlations. Acta Neuropatho 126(6):859-79

10. Mann DM et al (2013) Dipeptide repeat proteins are present in the p62 positive inclusions in patients with frontotemporal lobar degeneration and motor neurone disease associated with expansions in C90RF72. Acta Neuropathol Commun 1(1):68

11. Troakes C et al (2012) An MND/ALS phenotype associated with C9orf72 repeat expansion: abundant p62-positive, TDP-43-negative inclusions in cerebral cortex, hippocampus and cerebellum but without associated cognitive decline. Neuropathology 32(5):505-14

12. Al-Sarraj S et al (2011) p62 positive, TDP-43 negative, neuronal cytoplasmic and intranuclear inclusions in the cerebellum and hippocampus define the pathology of C9orf72-linked FTLD and MND/ALS. Acta Neuropathol 122(6):691-702

13. Gendron TF et al (2013) Antisense transcripts of the expanded C9ORF72 hexanucleotide repeat form nuclear RNA foci and undergo repeat-associated non-ATG translation in C9FTD/ALS. Acta Neuropathol 126(6):829-44

14. Davidson YS et al (2014) Brain distribution of dipeptide repeat proteins in frontotemporal lobar degeneration and motor neurone disease associated with expansions in C9ORF72. Acta Neuropathol Commun 2:70

15. Wen $X$ et al (2014) Antisense proline-arginine RAN dipeptides linked to C9ORF72-ALS/FTD form toxic nuclear aggregates that initiate in vitro and in vivo neuronal death. Neuron 84(6):1213-25

16. Yamakawa $\mathrm{M}$ et al (2015) Characterization of the dipeptide repeat protein in the molecular pathogenesis of c9FTD/ALS. Hum Mol Genet 24(6):1630-45

17. Tao $Z$ et al (2015) Nucleolar stress and impaired stress granule formation contribute to C9orf72 RAN translation-induced cytotoxicity. Hum Mol Genet 24(9):2426-41

18. Mackenzie IR, Frick P, Neumann M (2014) The neuropathology associated with repeat expansions in the C9ORF72 gene. Acta Neuropathol 127(3):347-57

19. Mori K et al (2013) Bidirectional transcripts of the expanded C9orf72 hexanucleotide repeat are translated into aggregating dipeptide repeat proteins. Acta Neuropathol 126(6):881-93

20. May S et al (2014) C9orf72 FTLD/ALS-associated Gly-Ala dipeptide repeat proteins cause neuronal toxicity and Unc119 sequestration. Acta Neuropathol 128(4):485-503

21. Zhang YJ et al (2014) Aggregation-prone c9FTD/ALS poly(GA) RAN-translated proteins cause neurotoxicity by inducing ER stress. Acta Neuropathol 128(4):505-24

22. Mizielinska S et al (2014) C9orf72 repeat expansions cause neurodegeneration in Drosophila through arginine-rich proteins. Science 345(6201):1192-4

23. Chew J et al (2015) C9ORF72 repeat expansions in mice cause TDP-43 pathology, neuronal loss, and behavioral deficits. Science 348:1151-4

\section{Submit your next manuscript to BioMed Central and take full advantage of:}

- Convenient online submission

- Thorough peer review

- No space constraints or color figure charges

- Immediate publication on acceptance

- Inclusion in PubMed, CAS, Scopus and Google Scholar

- Research which is freely available for redistribution

Submit your manuscript at www.biomedcentral.com/submit
C Biomed Central 